\title{
Research Article \\ Performance and Flow Field of a Gravitation Vortex Type Water Turbine
}

\author{
Yasuyuki Nishi and Terumi Inagaki \\ Department of Mechanical Engineering, Ibaraki University, 4-12-1 Nakanarusawa-cho, Hitachi-shi, Ibaraki 316-8511, Japan \\ Correspondence should be addressed to Yasuyuki Nishi; yasuyuki.nishi.fe@vc.ibaraki.ac.jp
}

Received 28 November 2016; Revised 15 February 2017; Accepted 5 March 2017; Published 16 March 2017

Academic Editor: Ryoichi Samuel Amano

Copyright (C) 2017 Yasuyuki Nishi and Terumi Inagaki. This is an open access article distributed under the Creative Commons Attribution License, which permits unrestricted use, distribution, and reproduction in any medium, provided the original work is properly cited.

\begin{abstract}
A gravitation vortex type water turbine, which mainly comprises a runner and a tank, generates electricity by introducing a flow of water into the tank and using the gravitation vortex generated when the water drains from the bottom of the tank. This water turbine is capable of generating electricity using a low head and a low flow rate with relatively simple structure. However, because its flow field has a free surface, this water turbine is extremely complicated, and thus its relevance to performance for the generation of electricity has not been clarified. This study aims to clarify the performance and flow field of a gravitation vortex type water turbine. We conducted experiments and numerical analysis, taking the free surface into consideration. As a result, the experimental and computational values of the torque, turbine output, turbine efficiency, and effective head agreed with one another. The performance of this water turbine can be predicted by this analysis. It has been shown that when the rotational speed increases at the runner inlet, the forward flow area expands. However, when the air area decreases, the backward flow area also expands.
\end{abstract}

\section{Introduction}

Many large-scale conventional hydraulic power generations mainly use medium- or high-heads and water turbines $[1,2]$ for conduits, such as the Francis water turbine. Recently, however, as public consciousness about renewable energies has risen, the demand for small-scale hydraulic power generation with a water turbine [3-7] for open channels has been increasing, with the use of so-far unused common rivers or waterways that have low heads and low flow rates.

Therefore, we focused on a water turbine used in the Gravitation Water Vortex Power Plant (GWVPP) [8], which generates electricity with a low head and a low flow rate. This gravitation vortex type water turbine mainly comprises a runner and a tank. On introducing a flow of water into the tank, the turbine generates electricity from the gravitation vortex that occurs while draining the water from the bottom of the tank. In addition, it is thought that this water turbine has an aeration function to raise the dissolved oxygen concentration of the downstream water by rolling up the air above the free surface around the runner. Despite the fact that this water turbine has a relatively simple structure, the flow field is extremely complicated because of its free surface. However, although some studies on other types of runners related to this kind of water turbine have been presented [9-12], their flow fields have not been investigated in detail. To improve the performance of the water turbine, it is important to study the flow field in detail in order to determine its relevance to the performance characteristic. Although a numerical analysis is effective for this, because this water turbine operates by using a gravitation vortex, it is necessary to conduct a numerical analysis with consideration to the free surface. Because a numerical analysis with consideration to the free surface requires a large computational load, there are few examples of where it has been applied to a water turbine. Recently, however, it has started to be applied to a spiral water turbine [13], an undershot cross-flow water turbine [14, 15], and a propeller water turbine $[16,17]$.

In light of this background, this study aims to clarify the performance of a gravitation vortex type water turbine and elucidate its flow field. We performed numerical analysis by considering the free surface, conducted a performance test and a visualization experiment, and verified the validity of our analysis. Furthermore, we examined the flow field around 

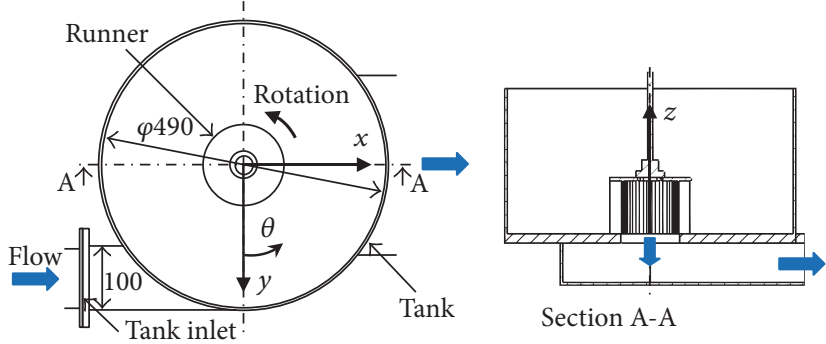

Section A-A

FIgURE 1: Test water turbine.
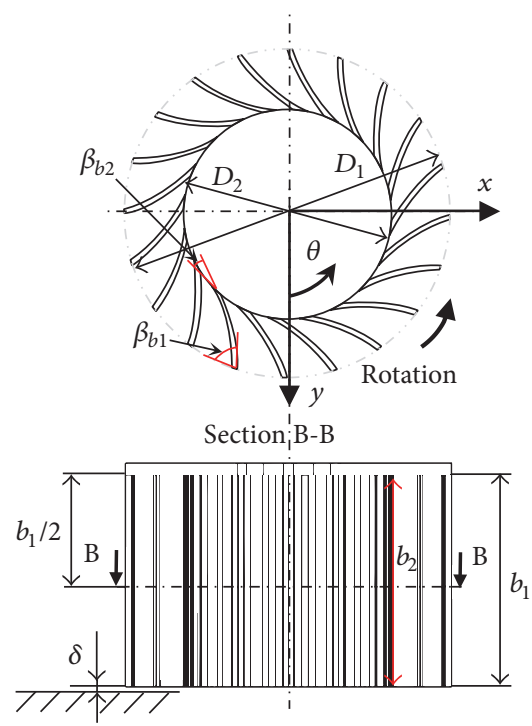

FIgURE 2: Runner.

the runner at the center of the blade width in detail using a numerical analysis.

\section{Experimental Apparatus and Methods}

2.1. Gravitation Vortex Type Water Turbine. An overview of the gravitation vortex type water turbine is shown in Figure 1. This water turbine mainly comprises a runner and a tank and generates electricity from the gravitation vortex that occurs in the tank when the water is drained. An overview of the runner is illustrated in Figure 2, and its specifications are given in Table 1. This runner has a centrifugal form, which is different from the form of the paddle-type runner that has been used in previous studies [9-12]. The blade inlet diameter (outer diameter) is $D_{1}=140 \mathrm{~mm}$, blade outlet diameter (inner diameter) is $D_{2}=90 \mathrm{~mm}$, blade inlet width is $b_{1}=91 \mathrm{~mm}$, blade outlet width is $b_{2}=91 \mathrm{~mm}$, and number of blades is $z=20$. Section B-B in Figure 2 is a section at the center of the blade width. The inner diameter of the cylindrical tank is $490 \mathrm{~mm}$, and the diameter of the hole at the bottom of tank is $100 \mathrm{~mm}$. In addition, the coordinate system is defined as shown in Figure 1. The circumferential angle $\theta$ is defined as $\theta=0^{\circ}$ on the positive $y$ axis, and its positive direction is counterclockwise.
TABLE 1: Specifications of runner.

\begin{tabular}{lc}
\hline Outer diameter: $D_{1}$ & $0.14 \mathrm{~m}$ \\
Inner diameter: $D_{2}$ & $0.09 \mathrm{~m}$ \\
Inlet width: $b_{1}$ & $0.091 \mathrm{~m}$ \\
Outlet width: $b_{2}$ & $0.091 \mathrm{~m}$ \\
Inlet angle: $\beta_{b 1}$ & $71.9^{\circ}$ \\
Outlet angle: $\beta_{b 2}$ & $19.0^{\circ}$ \\
Tip clearance: $\delta$ & $0.5 \mathrm{~mm}$ \\
Number of blades: $Z$ & 20 \\
\hline
\end{tabular}

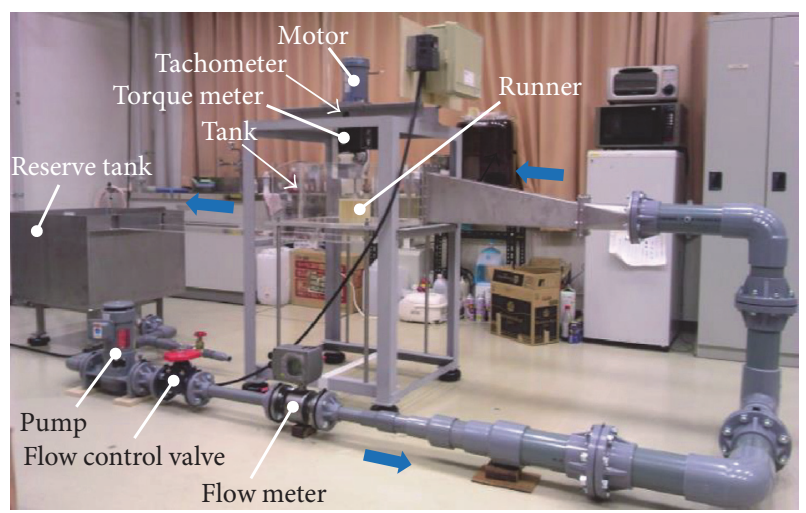

FIGURE 3: Experimental apparatus.

2.2. Experimental Apparatus and Methods. An overview of the experimental apparatus is shown in Figure 3. The flow rate $Q$ of water supplied by the pump was measured with an electromagnetic flow meter (Toshiba Corporation; LF620). The experiment was conducted under the condition of constant flow rate $Q=0.00285 \mathrm{~m}^{3} / \mathrm{s}$. The load to the runner was controlled by a motor and an inverter, and the rotational speed was arbitrarily set. The rotational speed $n$ and torque $T$ were measured with a magnetic rotation detector (Ono Sokki Co., Ltd.; MP-981) and a torque detector (Ono Sokki Co., Ltd.; SS-005), respectively. The turbine output $P$ was obtained by

$$
P=\frac{2 \pi n T}{60} .
$$

Here the torque $T$ was corrected by measuring the idling torque without the runner. The effective head $H$ is defined by the following as shown in Figure 4.

$$
H=h^{\prime}+h_{3}+\frac{v_{3}^{2}}{2 g}-h_{4}-\frac{v_{4}^{2}}{2 g} .
$$

Here the upstream water depth $h_{3}$ was measured at the tank inlet in the vicinity of the wall surface on the $+y$-axis with a ruler. The downstream water depth $h_{4}$ was measured by a point gauge (Kenek Corporation; $\mathrm{PH}-102$ ) at five points from the vicinity of the wall surface on the $+y$-axis to the center at the position of $6 D_{1}$ downstream from the downstream atmospheric opening from which the average water depth 


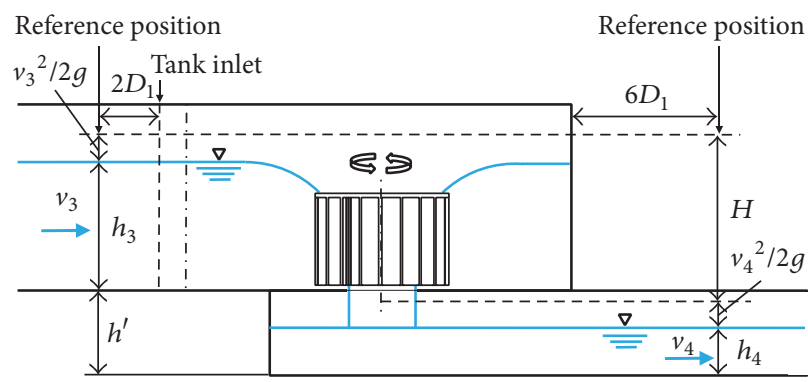

FIGURE 4: Definition of performance evaluation.

was obtained. The upstream velocity $v_{3}$ and downstream velocity $v_{4}$ were calculated by

$$
\begin{aligned}
& v_{3}=\frac{Q}{B_{3} h_{3}}, \\
& v_{4}=\frac{Q}{B_{4} h_{4}} .
\end{aligned}
$$

Here $B_{3}$ and $B_{4}$ are the waterway widths of the tank inlet and downstream, respectively. In addition, the turbine efficiency $\eta$ was calculated by

$$
\eta=\frac{P}{\rho g Q H} .
$$

A digital camera (Casio Computer Co., Ltd.; EXILIM EXF1) was used to visualize the flow field at a frame rate of 30 frames per second (fps).

\section{Numerical Analysis Method and Conditions}

In this study, three-dimensional unsteady flow analysis was performed by considering the free surface. The generalpurpose thermal fluid analysis software, ANSYS CFX15.0 (ANSYS, Inc.), was used for the calculations. Moreover, the volume of fluid (VOF) method [18], which is suitable for a flow field that has a clear interface between two phases and expresses the actual performance [13-17] in a free surface flow analysis of a water turbine, was also used. The working fluids were water and air. The governing equations were the mass conservation equation, momentum conservation equation, and volume conservation equation. A guideline for using a turbulence model that is suitable for the VOF method has not been clarified. Therefore, the shear stress transport (SST) model [19], which can model the actual performance [13, 16, 17] of a free surface flow analysis of a water turbine using the VOF method, has been used as the turbulence model.

The entire area of calculation is shown in Figure 5. This is divided into four main areas: runner, tank, upstream waterway, and downstream waterway. The downstream waterway is $10 D_{1}$ in length from the atmospheric opening to the outlet boundary. The reference position of the upstream side was set to the tank inlet, which was the same as that in the experiment. The reference position of the downstream side was set to $6 D_{1}$ downstream from the atmospheric opening

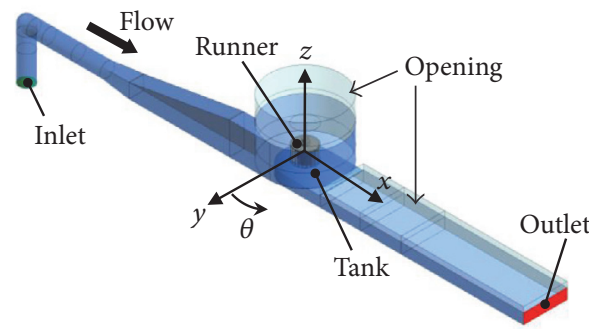

Figure 5: Calculating area.

of the downstream waterway. At these reference positions, the distribution of each water depth was obtained in the width direction, assuming that the water surface is equivalent to that of a water volume fraction of 0.5. The upstream water depth $h_{3}$ and downstream water depth $h_{4}$ are the averages obtained from the distribution of water depth in the width direction. As an example, the grids used in the runner and tank calculations are illustrated in Figures 6(a) and 6(b), respectively. The runner, tank, upstream waterway, and downstream waterway have approximately $446,000,541,000$, 434,000 , and 780,000 computational elements, respectively, which totals to $2,201,000$. Computational grids $[14,15]$ using an undershot cross-flow water turbine were prepared, as these are able to verify the free surface flow analysis and the experiments of the flow field relatively well. As boundary conditions, the mass flow rate $(2.838 \mathrm{~kg} / \mathrm{s})$ was given to the inlet boundary, an open boundary (total pressure of $0 \mathrm{~Pa}$ for the inflow, or relative static pressure of $0 \mathrm{~Pa}$ for the outflow) to the outlet boundary, and an arbitrary rotational speed to the runner. In addition, the upper surfaces of the tank and downstream waterway were set to open boundaries (relative static pressure of $0 \mathrm{~Pa}$ ) so that air could enter and exit freely. The wall surface was set to the no-slip condition. With reference to the calculations, a steady flow analysis was first conducted, followed by an unsteady flow analysis using the steady flow results as the initial conditions. In the unsteady flow analysis, the boundary between a rotational and a stationary area was connected using the transient rotor-stator method. The calculation continued until the flow became almost stable, as determined by its fluctuations. A total of 180 time steps were used, during which the runner completes one rotation.

\section{Results and Discussion}

4.1. Comparison of Water Turbine Performance. A comparison between the experimental and calculation results for this water turbine in relation to its performance is shown in Figures 7(a) and 7(b). It is observed that the experimental and calculation results are in good agreement in terms of torque $T$, turbine output $P$, turbine efficiency $\eta$, and effective head $H$. As the rotational speed $n$ is increased, the torque $T$ decreases and the effective head $H$ increases marginally. The reasons for this appear to be that the increase of effective head $H$ derives mainly from the increase of upstream water depth $h_{3}$ and that the resistance of the runner increases with the increase 


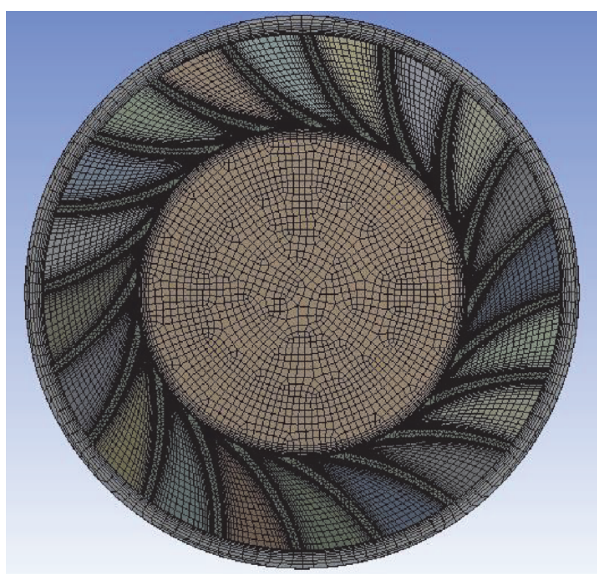

(a) Runner

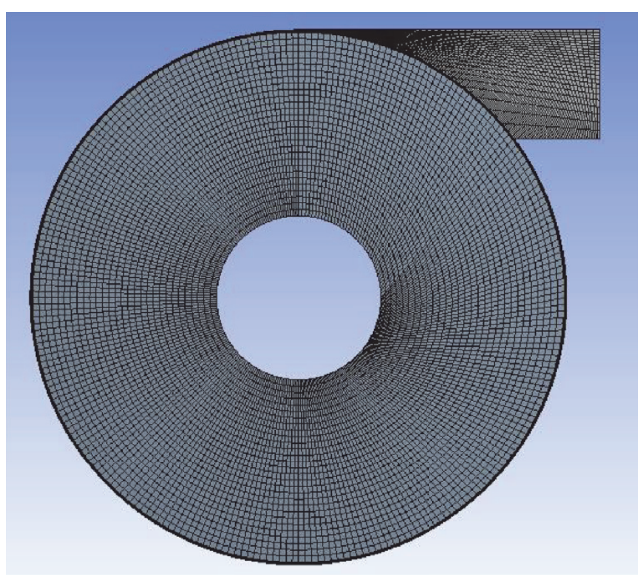

(b) Tank

FIgURE 6: Computational grids.

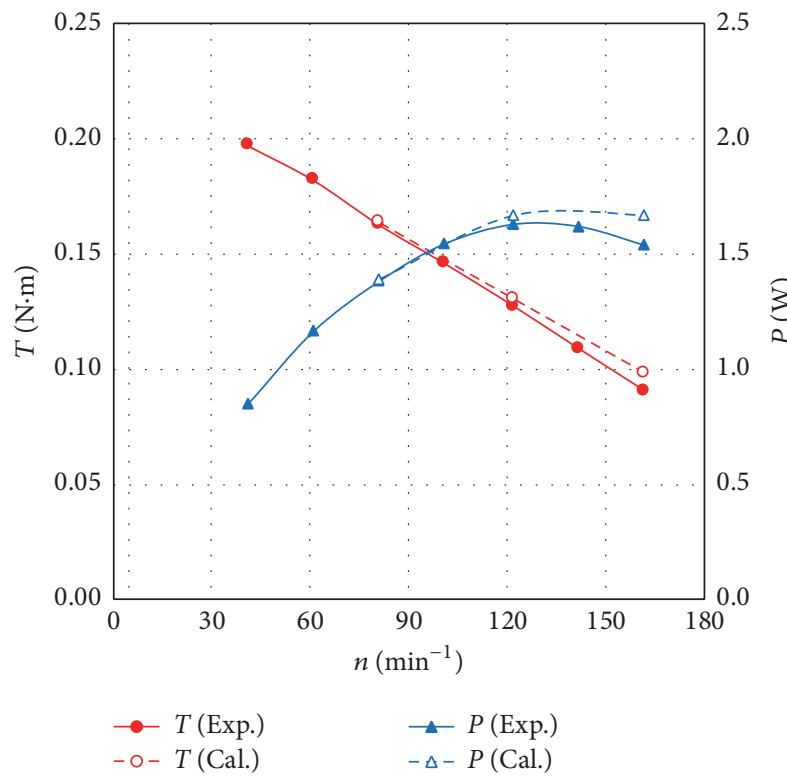

(a) Torque and turbine output

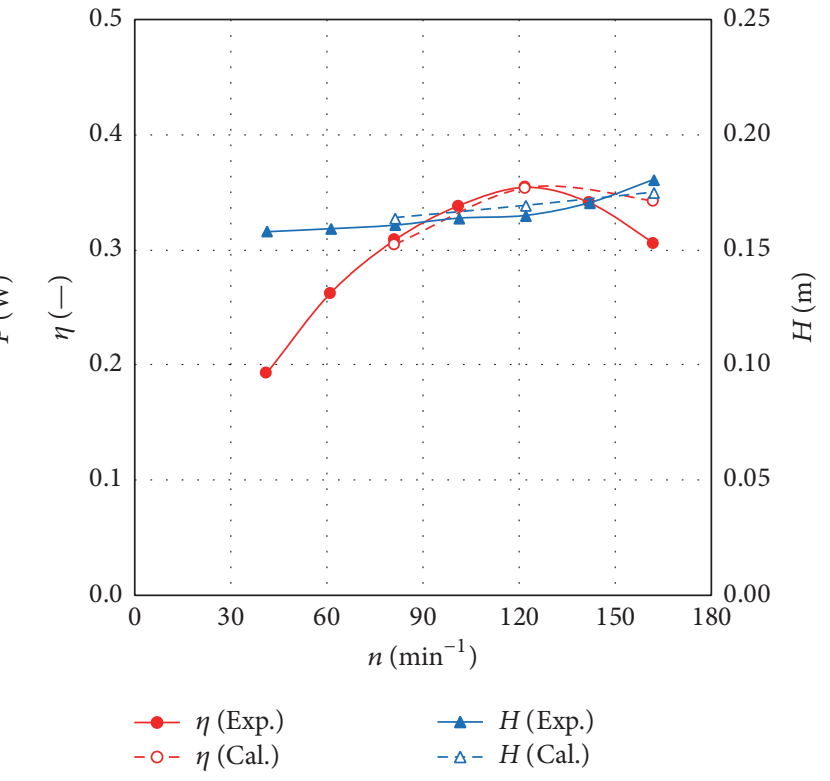

(b) Turbine efficiency and effective head

Figure 7: Turbine performance.

of rotational speed $n$. Both turbine output $P$ and turbine efficiency $\eta$ show maximum values at the rotational speed $n=122 \mathrm{~min}^{-1}$. The maximum experimentally determined efficiency is approximately 0.354 at a specific speed $n_{s}$ of approximately $47\left[\mathrm{~min}^{-1}, \mathrm{~kW}, \mathrm{~m}\right]$.

4.2. Comparison of Free Surface Flow Field. The experimental results for the free surface flow field of this water turbine are shown in Figures $8(\mathrm{a})-8(\mathrm{c})$, and the calculation results are shown in Figures 9(a)-9(c). In these calculation results, the water/air boundary is defined as $\mathrm{VF}=0.5$, and this isosurface is illustrated. The water depth around the runner varies in the circumferential direction in both the experimental and calculation results, becoming lower in the $+x$ direction. In addition, the water depth varies greatly according to the rotational speed. The experimentally determined free surface shape with change in the rotational speed can be seen to agree qualitatively with the calculation results. Because the flow speed depends on the water depth in the circumferential direction, if it is combined with a change of operational conditions such as the rotational speed, it is presumed that the flow field around the runner will become extremely complicated. Therefore, in this study, we next discuss the flow field at the center of the blade width (section B-B in Figure 1).

4.3. Flow Field at the Center of the Blade Width. First, in order to identify the water/air interface, the circumferential distribution of water volume fraction $\mathrm{VF}_{1}$ obtained numerically 


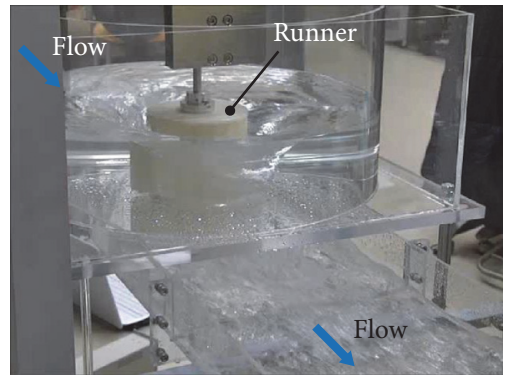

(a) $n=81 \mathrm{~min}^{-1}$

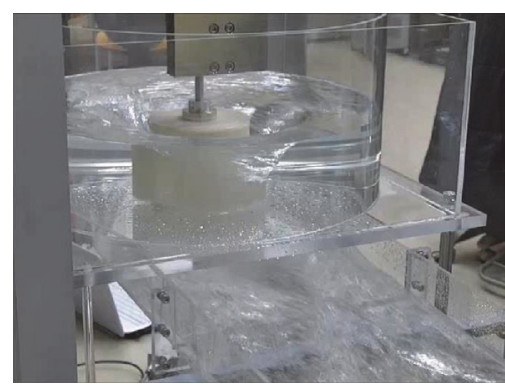

(b) $n=122 \mathrm{~min}^{-1}$

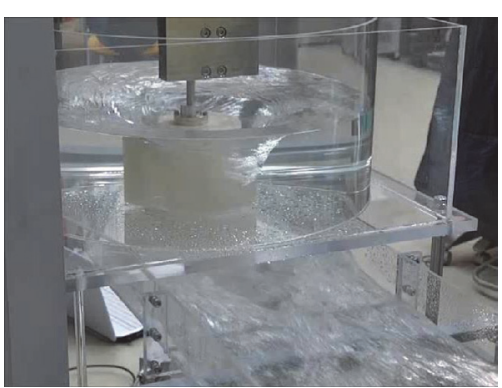

(c) $n=162 \mathrm{~min}^{-1}$

Figure 8: Flow field by experiment.

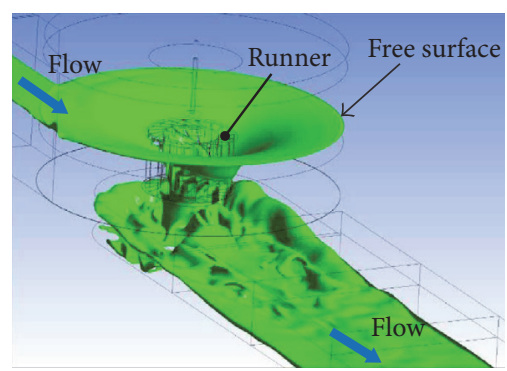

(a) $n=81 \mathrm{~min}^{-1}$

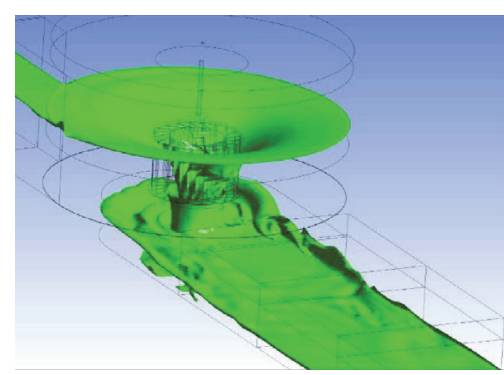

(b) $n=122 \mathrm{~min}^{-1}$

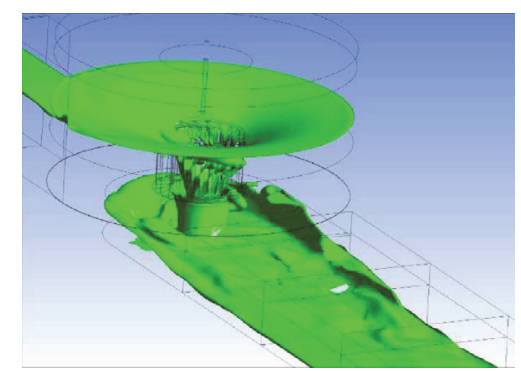

(c) $n=162 \mathrm{~min}^{-1}$

FIgURE 9: Flow field by calculation.

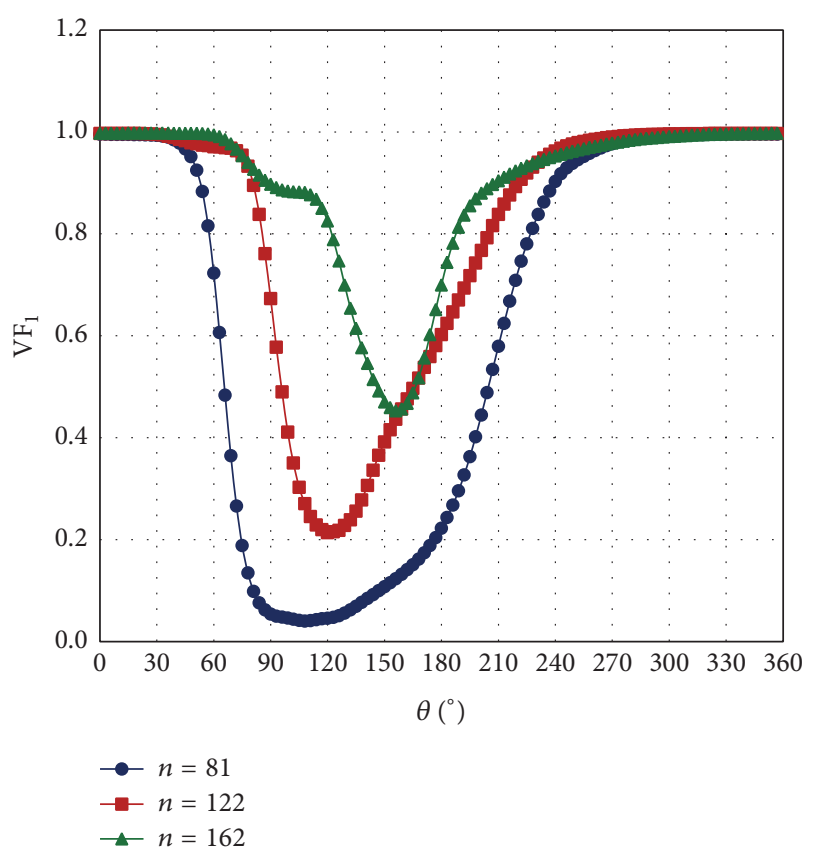

Figure 10: Volume fraction of water at runner inlet (Cal.).

for a runner inlet at the center of the blade width is shown in Figure 10. Here $\mathrm{VF}_{1}$ is the time average value during one rotation of the runner. Air is represented by $0 \leq \mathrm{VF}<0.5$, water is represented by $0.5<\mathrm{VF} \leq 1$, and the interface between them is represented by $\mathrm{VF}=0.5$. These notations are the
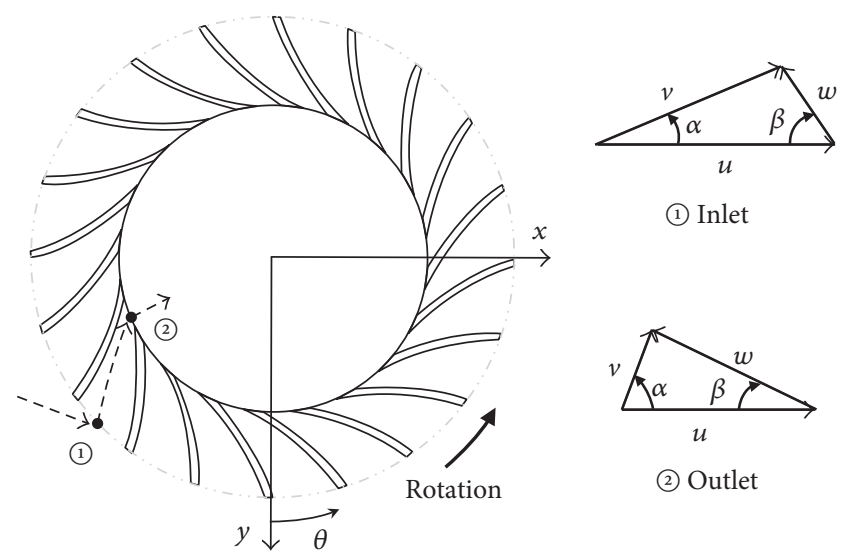

(1) Inlet

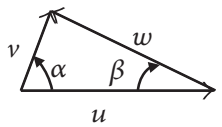

(2) Outlet

FIGURE 11: Velocity triangles.

same as those of a runner outlet, which will be described subsequently. According to Figure 10, at the maximumefficiency rotational speed $n=122 \mathrm{~min}^{-1}$, the water area of $\mathrm{VF}>0.5$ is at $\theta=0^{\circ}-93^{\circ}$ and $\theta=168^{\circ}-360^{\circ}$, and thus its total effective angle is approximately $285^{\circ}$. This water area reduces with decrease in the rotational speed and increases with increase of it. This appears to be because the resistance of the runner increases with increase in rotational speed, as described before.

Here velocity triangles of the water turbine are illustrated in Figure 11. The velocity $v_{r}$ is defined in the radially inward direction, $v_{u}$ in the rotation direction, and $v_{a}$ in the $+z$ 


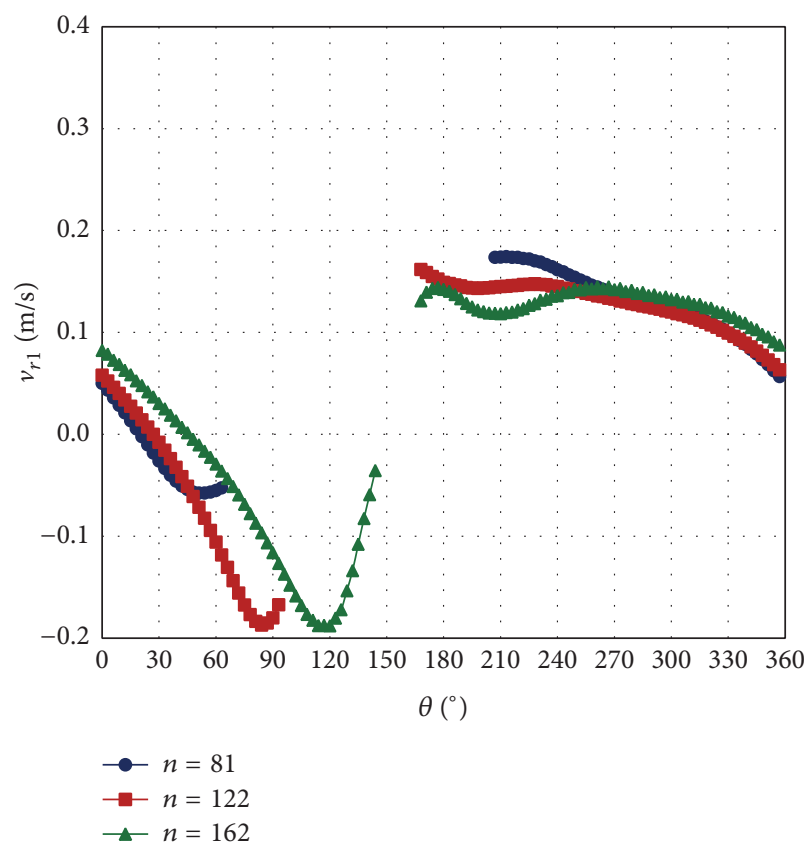

(a) Radial component

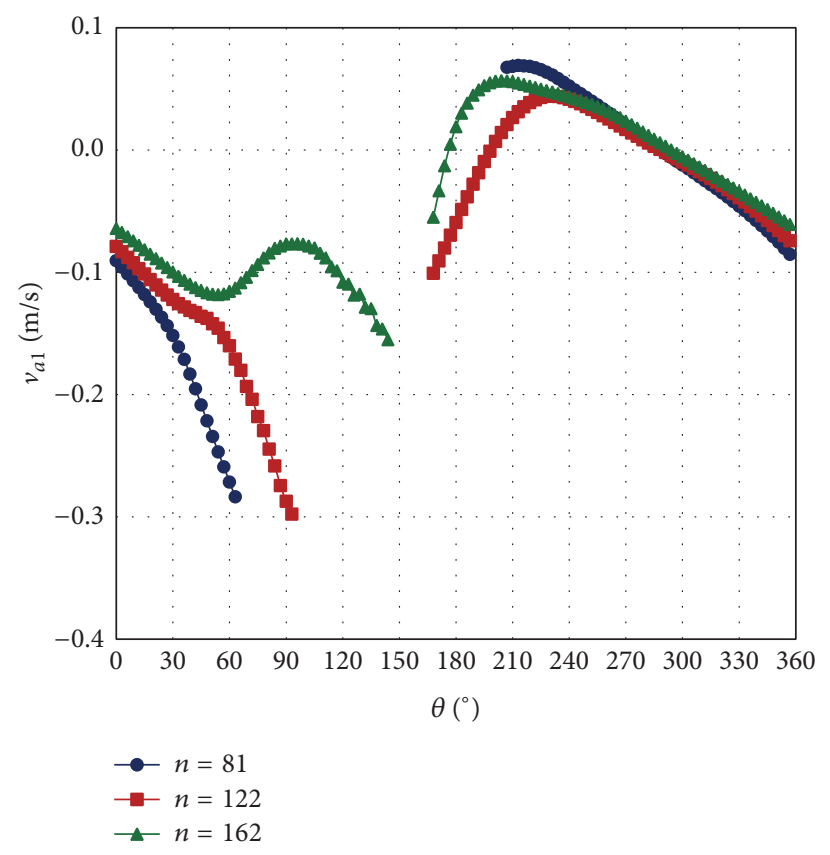

(b) Axial component

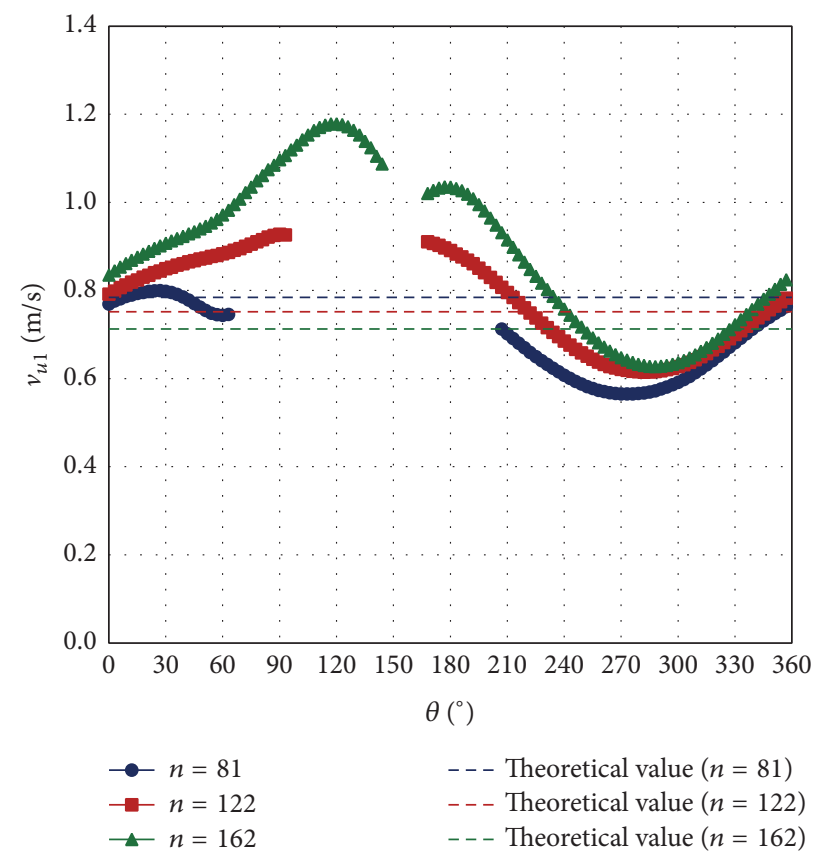

(c) Circumferential component

Figure 12: Absolute velocity at runner inlet (Cal.).

direction. Figures $12(\mathrm{a})-12(\mathrm{c})$ show circumferential distributions of the radial component $v_{r 1}$, axial component $v_{a 1}$, and circumferential component $v_{u 1}$ of the absolute velocity of a runner inlet at the center of the blade width in the numerical analysis. Here each component is the time average value during one rotation of the runner and displays a water area identified only from the water volume fraction. These notations are the same as those of a runner outlet, which will be described subsequently. The theoretical $v_{u 1}$ value obtained from the following expression that is based on the assumption that the tank has a free vortex type flow is also shown in Figure 12(c):

$$
v_{u 1}=\frac{r_{3} v_{3}}{r_{1}},
$$

where $r_{1}$ is the outer radius of the runner inlet and $r_{3}$ is the representative radius of the tank inlet. The radius $(70.5 \mathrm{~m})$ at the measuring point on the periphery of the runner inlet and the radius $(245 \mathrm{~mm})$ in the tank were used as $r_{1}$ and $r_{3}$, respectively. The value obtained from (3) was used as $v_{3}$. 
From Figure 12(a), it can be observed that $v_{r 1}$ is not uniform in the circumferential direction at any rotational speed. For $n=122 \mathrm{~min}^{-1}$, the forward flow area in which $v_{r 1}$ is positive appears at $\theta=0^{\circ}-24^{\circ}$ and $\theta=168^{\circ}-360^{\circ}$; its total effective angle is approximately $216^{\circ}$. In addition, the backward flow area appears at $\theta=27^{\circ}-93^{\circ}$; its effective angle is approximately $66^{\circ}$. In this backward flow area, $v_{a 1}$ is negatively large, as is shown in Figure 12(b). With increase in the rotational speed, the forward flow area increases, but because of the reduction in the air area, the backward flow area also increases. Moreover, with increase in the rotational speed, the value of $\theta$ at which $v_{r 1}$ changes from positive to negative shifts to the large $\theta$ side. In Figure 12(c), although the computational and theoretical $v_{u 1}$ values are relatively similar at $\theta=0^{\circ}$ near the tank inlet, they differ in other $\theta$ regions. Although the theoretical $v_{u 1}$ value decreases as the rotational speed increases, the computational $v_{u 1}$ value increases as the rotational speed increases, and it becomes large at both ends of the air area at any rotational speed. Because the water area for low values of $\theta$ of the air area is a backward flow area, the water area for large values of $\theta$ of the air area is considered to greatly contribute to the conversion of energy. For the circumferential velocity, $u_{1}$, at the runner inlet, $u_{1}=$ $0.594 \mathrm{~m} / \mathrm{s}$ at $n=81 \mathrm{~min}^{-1}, u_{1}=0.894 \mathrm{~m} / \mathrm{s}$ at $n=122 \mathrm{~min}^{-1}$, and $u_{1}=1.188 \mathrm{~m} / \mathrm{s}$ at $n=162 \mathrm{~min}^{-1}$. At $n=122$ and $162 \mathrm{~min}^{-1}$, $v_{u 1}$ at both ends of the air area is nearly the same as $u_{1}$. Therefore, a flow in the tank of this water turbine is not a perfect free vortex, and it is greatly influenced by the rotation of the runner near the runner inlet. Because this water turbine does not have guide vane upstream of the runner, a uniform and strong circumferential spiral flow can be produced by designing the tank shape that improves the turbine output.

The numerically determined circumferential distribution of the relative flow angle $\beta_{1}$ of a runner inlet at the center of the blade width is shown in Figure 13. At $n=81 \mathrm{~min}^{-1}$, the relative flow angle $\beta_{1}$ for $\theta=240^{\circ}-300^{\circ}$ shows a relatively close value at the blade inlet angle $\beta_{b 1}=71.9^{\circ}$. However, at $n$ $=122$ and $n=162 \mathrm{~min}^{-1}$, the relative flow angle $\beta_{1}$ dissociates greatly from the blade inlet angle, and the shock loss appears to increase. Therefore, it is necessary to control the flow in the tank and homogenize the relative flow angle $\beta_{1}$ in the circumferential direction in order to decrease the shock loss at the blade inlet.

The numerically determined circumferential distribution of water volume fraction $\mathrm{VF}_{2}$ of a runner outlet at the center of the blade width is shown in Figure 14. At $n=122 \mathrm{~min}^{-1}$, the largest water area is for $\theta=0^{\circ}-15^{\circ}$ and $\theta=228^{\circ}-360^{\circ}$; the total effective angle is approximately $147^{\circ}$. This water area is considerably smaller than that of a runner inlet and does not change with the rotational speed.

Figures 15(a)-15(c) show the numerically determined time average values of the radial component $v_{r 2}$, axial component $v_{a 2}$ and circumferential component $v_{u 2}$ of the absolute velocity of a runner outlet at the center of the blade width. According to Figure 15(a), $v_{r 2}$ distribution at each rotational speed is almost the same and does not show a backward flow at any rotational speed. From Figure 15(b), $v_{a 2}$ component of the water area at the large $\theta$ side of the air area decreases

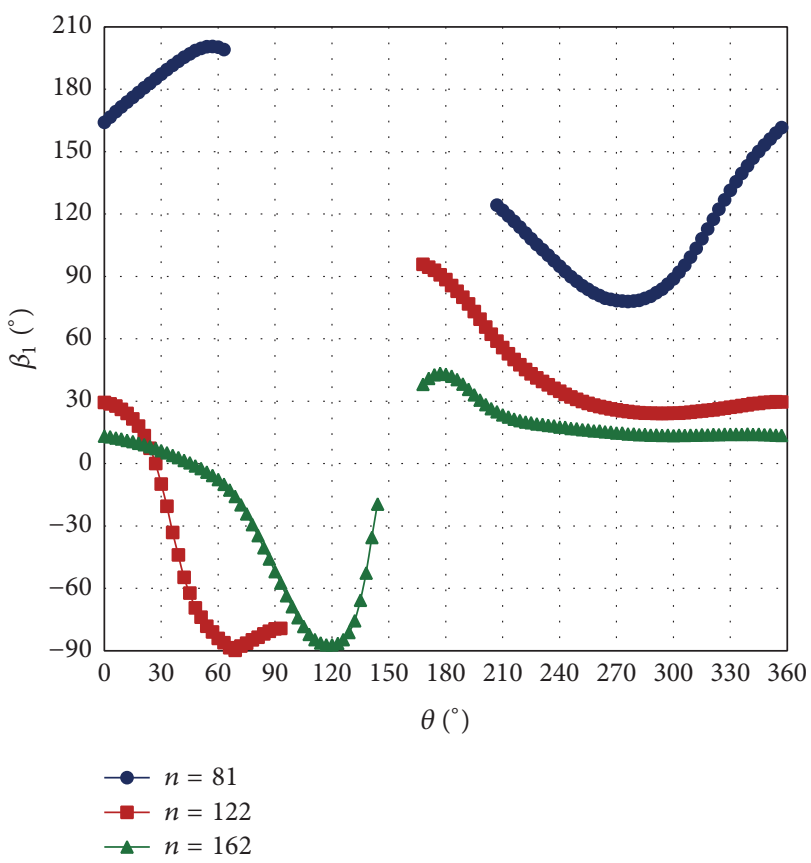

FIgURE 13: Relative flow angle at runner inlet (Cal.).

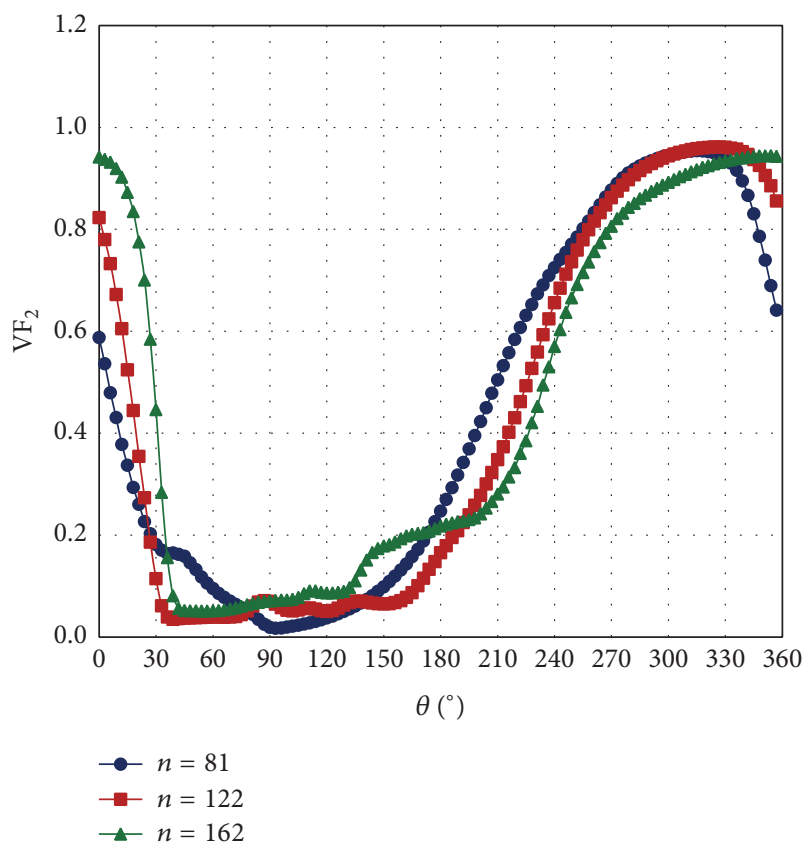

FIgURE 14: Volume fraction of water at runner outlet (Cal.).

rapidly as $\theta$ increases. From Figure 15(c), it can be observed that at $n=122 \mathrm{~min}^{-1}, v_{u 2}$ is the largest around $\theta=15^{\circ}$, which shows the remains of the rotational component but is relatively small for $\theta=228^{\circ}-285^{\circ}$. However, for this range of $\theta$, a negative rotation remains at $n=81 \mathrm{~min}^{-1}$ and a positive rotation remains at $n=162 \mathrm{~min}^{-1}$.

The flow rate and the angular momentum per unit time that flow in and out at the runner inlet and outlet relates to the torque of a water turbine studied. 


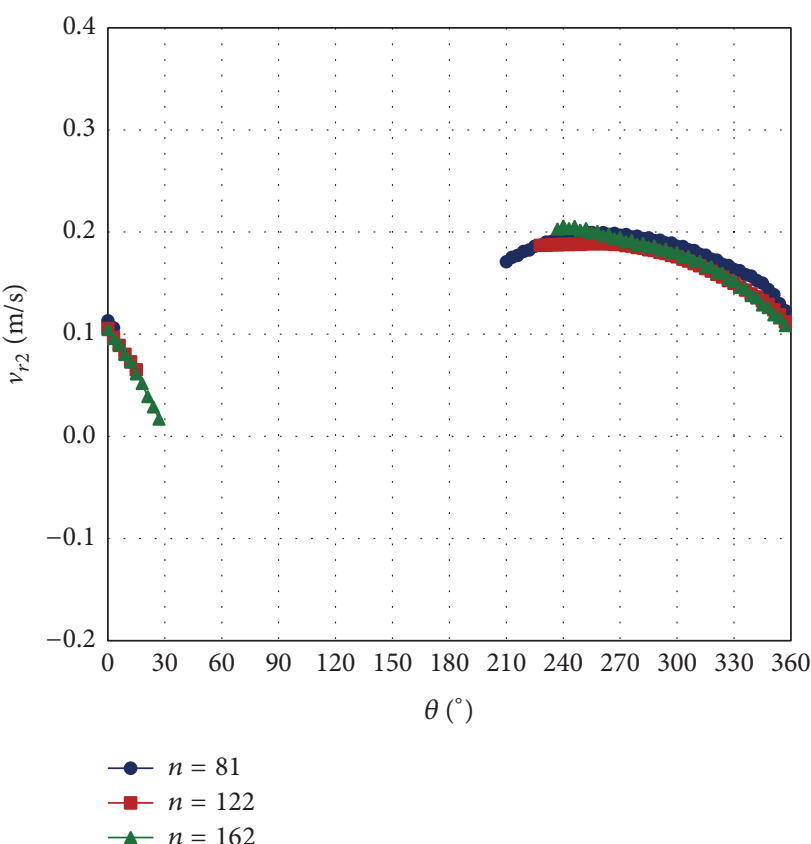

(a) Radial component

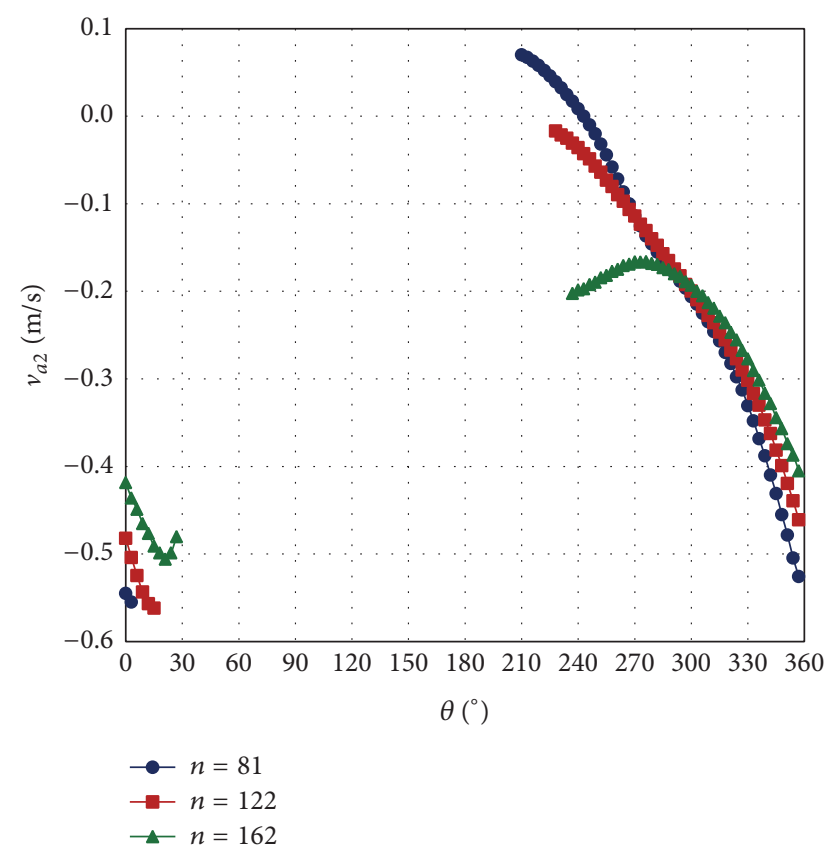

(b) Axial component

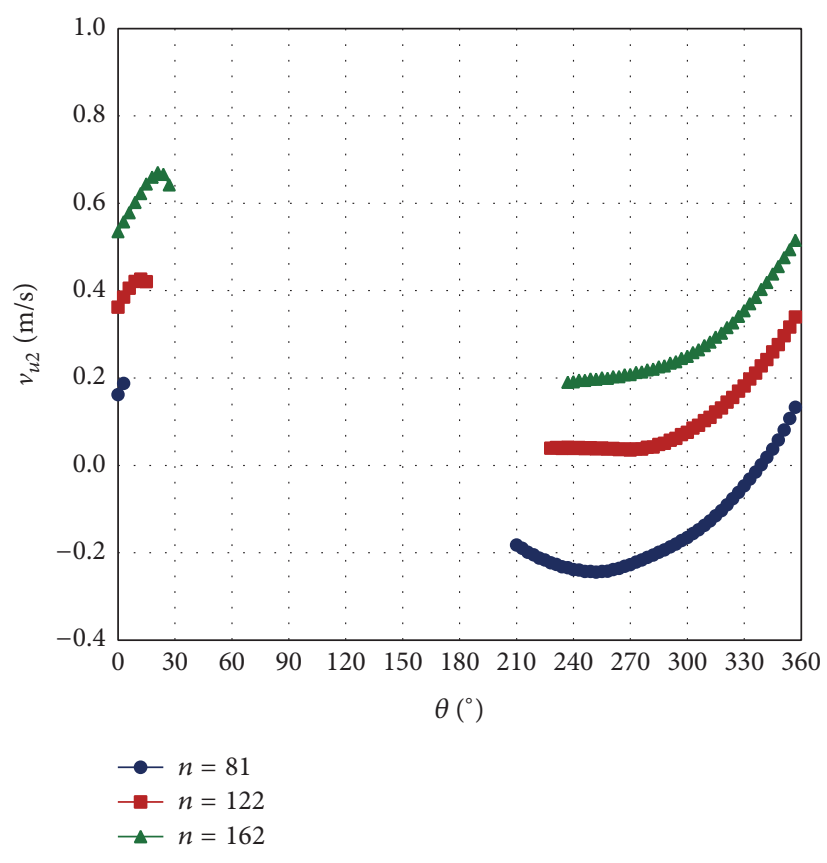

(c) Circumferential component

FIgURe 15: Absolute velocity at runner outlet (Cal.).

The flow rates $q_{1}$ and $q_{2}$ per unit blade width $(1 \mathrm{~mm})$ at the runner inlet and outlet are expressed as the following equations:

$$
\begin{aligned}
& q_{1}=\frac{2 \pi r_{1} \overline{v_{r 1}}}{1000}, \\
& q_{2}=\frac{2 \pi r_{2} \overline{v_{r 2}}}{1000} .
\end{aligned}
$$

$\overline{v_{r 1}}$ and $\overline{v_{r 2}}$ were obtained from the following equations by using the time average values of $v_{r 1}$ and $v_{r 2}$ during one rotation of the runner at each measuring point.

$$
\begin{aligned}
& \overline{v_{r 1}}=\frac{1}{\theta_{w}} \int_{\theta_{w}} v_{r 1} d \theta_{w}, \\
& \overline{v_{r 2}}=\frac{1}{\theta_{w}} \int_{\theta_{w}} v_{r 2} d \theta_{w} .
\end{aligned}
$$

Here $\theta_{w}$ is the circumferential angle of the water area. 


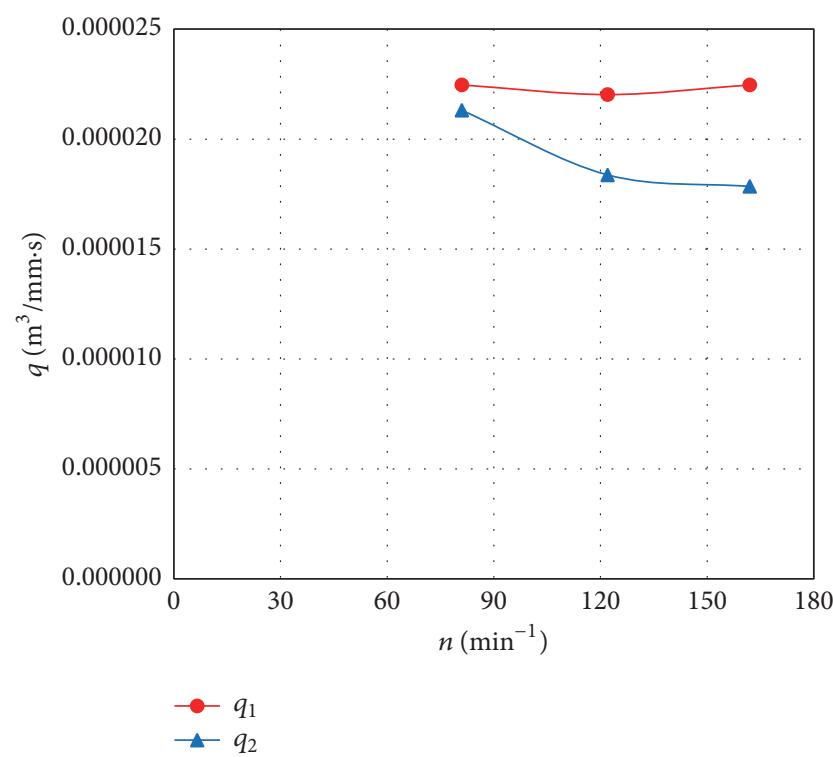

FIGURE 16: Flow rate per unit blade width.

Therefore, the angular momentums, $L_{1}$ and $L_{2}$, per unit blade width $(1 \mathrm{~mm})$ and unit time at the runner inlet and outlet can be expressed as the following equations:

$$
\begin{aligned}
& L_{1}=\rho q_{1} r_{1} \overline{\overline{v_{u 1}}}, \\
& L_{2}=\rho q_{2} r_{2} \overline{\overline{v_{u 2}}} .
\end{aligned}
$$

$\overline{\overline{v_{u 1}}}$ and $\overline{\overline{v_{u 2}}}$ were obtained from the following equations by using the time average values of $v_{r 1} v_{u 1}$ and $v_{r 2} v_{u 2}$ during one rotation of the runner at each measuring point.

$$
\begin{aligned}
& \overline{\overline{v_{u 1}}}=\frac{1}{\overline{v_{r 1}} \theta_{w}} \int_{\theta_{w}} v_{r 1} v_{u 1} d \theta_{w}, \\
& \overline{\overline{v_{u 2}}}=\frac{1}{\overline{v_{r 2}} \theta_{w}} \int_{\theta_{w}} v_{r 2} v_{u 2} d \theta_{w} .
\end{aligned}
$$

The relationship between the rotational speed, $n$, at the center of the blade width and the flow rates, $q_{1}$ and $q_{2}$, per unit blade width are shown in Figure 16. The relationships between the rotational speed, $n$, and the angular momentums, $L_{1}$ and $L_{2}$, per unit blade width and unit time are shown in Figure 17.

In Figure 16, although $q_{1}$ is rather small at $n=122 \mathrm{~min}^{-1}$, it is nearly constant when the rotational speed changes. Therefore, when the rotational speed increases, the forward flow area expands. However, as previously stated, the flow rate that flows in from the center of blade width barely changes. Conversely, $q_{2}$ is relatively similar to $q_{1}$ at $n=81 \mathrm{~min}^{-1}$. When the rotational speed increases, $q_{2}$ at $n=81 \mathrm{~min}^{-1}$ is approximately $20.6 \%$ lower than $q_{1}$. Since $v_{a 2}$ is a negative value, as stated above, the flow through the runner comes close to the tip side (bottom of the tank). Therefore, in order to design a high-performance runner, it is necessary to study the

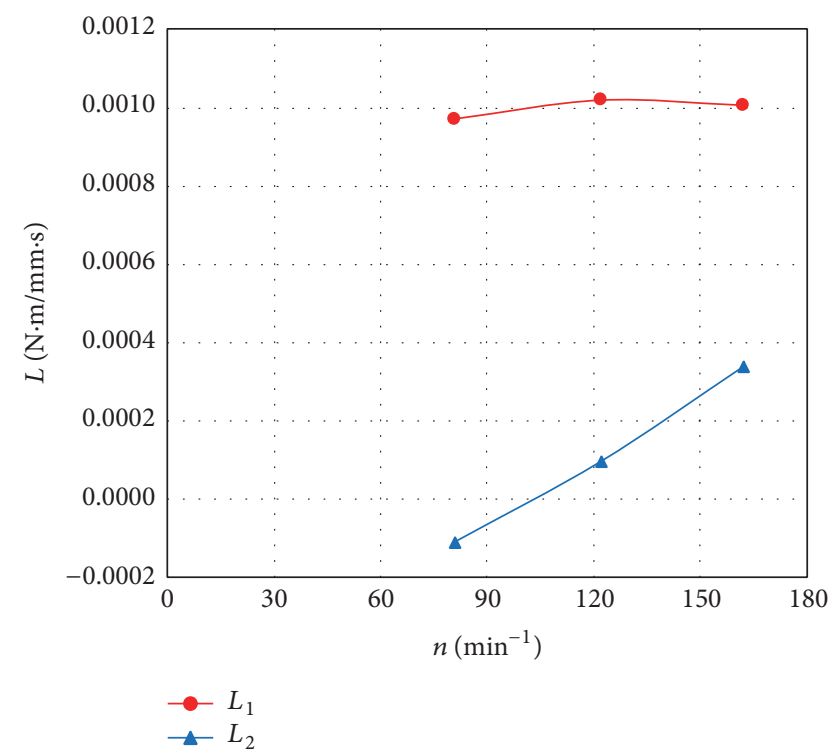

FIGURE 17: Angular momentum per unit blade width and unit time.

three-dimensional flow, including the direction of the blade width.

In Figure 17, although $L_{1}$ is rather large at $n=122 \mathrm{~min}^{-1}$, it is nearly constant when the rotational speed changes. However, $L_{2}$ has a large negative value at $n=81 \mathrm{~min}^{-1}$ and a large positive value at $n=162 \mathrm{~min}^{-1}$. When only the center of the blade width is considered, because the difference between $L_{1}$ and $L_{2}$ is the theoretical torque of the water turbine, change in torque when the rotational speed changes shows that difference of the angular momentum that remains at the runner outlet has a large influence. Since both the positive and negative angular momentums at the runner outlet cause an increase in the loss of waste, they are considered to be one of the factors related to the decrease in efficiency at low or high rotational speeds.

Figures 18(a)-18(c) illustrate the relative velocity vectors and water volume fraction of the runner. The cross section represents the center of the blade width (section B-B). It can be seen that with increase in the rotational speed at a runner inlet, as described above, the air area reduces but the backward flow area increases. In addition, at $n=$ $81 \mathrm{~min}^{-1}$, the relative water flow is relatively smooth along the blade, but, at $n=122$ and $162 \mathrm{~min}^{-1}$, it flows in at small angles.

\section{Conclusions}

The following matters were determined by our research of the performance of a gravitation vortex type water turbine and the flow field at the center of blade width through experiments and free surface flow analysis:

(1) The experimental and computational values of the torque, turbine output, turbine efficiency, and effective head agree well with one another. Thus, the 


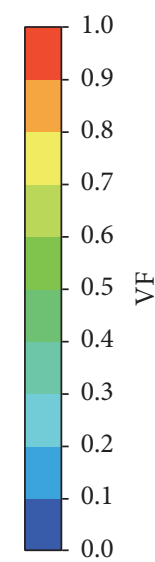

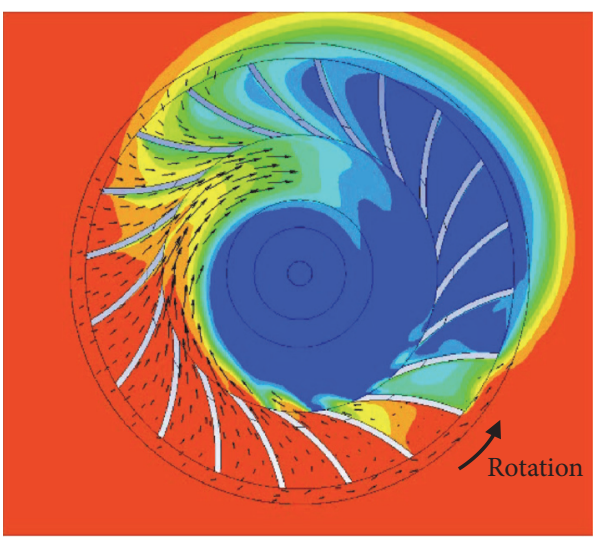

(a) $n=81 \mathrm{~min}^{-1}$

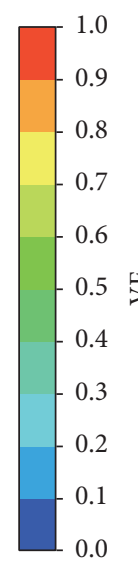

1.0
0.9
0.8
0.7
0.6
0.5
0.4
-0.3
0.2
0.1
0.0

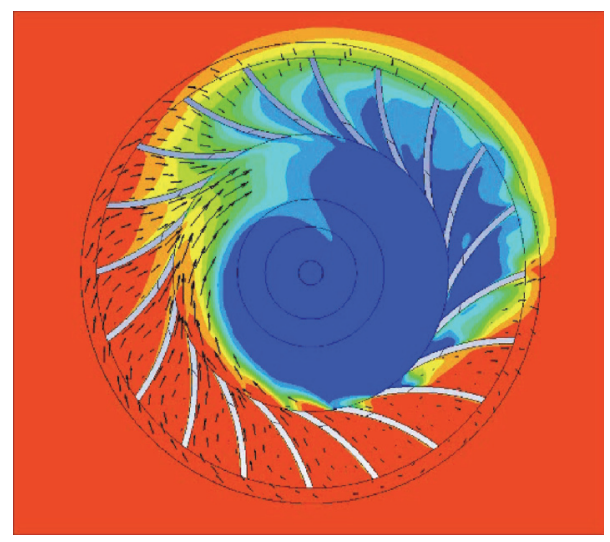

(b) $n=122 \mathrm{~min}^{-1}$

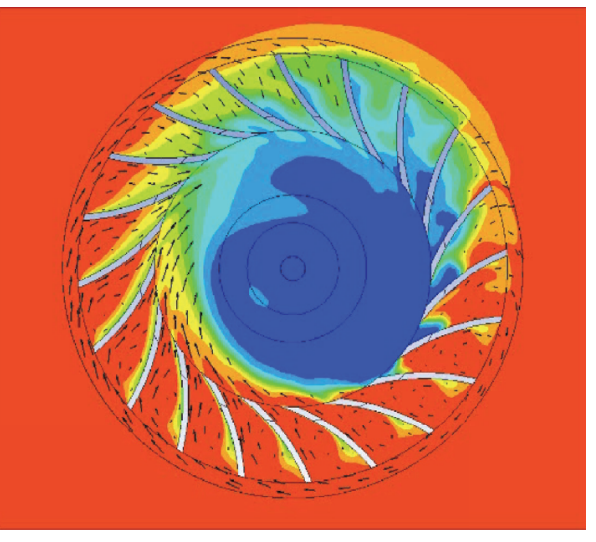

(c) $n=162 \mathrm{~min}^{-1}$

FIGURE 18: Relative velocity vectors and volume fraction of water (Cal.).

performance of this water turbine can be predicted by this analysis.

(2) With increase in the rotational speed at a runner inlet, the forward flow area increases, as does the backward flow area because of the reduction in the air area. However, the flow rate that flows in from the center of the blade width barely changes.

(3) The flow in the tank of this water turbine is not a perfect free vortex, and it is greatly influenced by the rotation of the runner near the runner inlet.

(4) The water area of a runner outlet is considerably smaller than that of a runner inlet and does not change with the rotational speed. In addition, backward flow does not occur at a runner outlet.

(5) When the rotational speed changes, the angular momentum per unit time that flows from the runner inlet is nearly constant. The angular momentum per unit time that flows from the runner outlet shows a large negative value at low-speed rotations and a large positive value at high-speed rotations. It also has a large influence on the torque when the rotational speed changes.

\section{Nomenclature}

$b$ : Blade width $\mathrm{m}$

$B$ : Waterway width $\mathrm{m}$

$D$ : Runner diameter $m$

g: Gravitational acceleration $\mathrm{m} / \mathrm{s}^{2}$

$h$ : Water depth $\mathrm{m}$

$h^{\prime}$ : The difference in height between the bottom surface of tank and the bottom surface of downstream waterway $\mathrm{m}$

$H$ : Effective head $\mathrm{m}$ $\left(=h^{\prime}+h_{3}+v_{3}^{2} / 2 g-h_{4}-v_{4}^{2} / 2 g\right)$

$L$ : Angular momentum per unit blade width and unit time $\mathrm{N} \cdot \mathrm{m} / \mathrm{mm} \cdot \mathrm{s}$

$n$ : Rotational speed $\mathrm{min}^{-1}$

$n_{s}:$ Specific speed $\min ^{-1}, \mathrm{~kW}, \mathrm{~m}$ $\left(=n(P / 1000)^{1 / 2} / H^{5 / 4}\right)$

$P$ : Turbine output $\mathrm{W}(=2 \pi n T / 60)$

$q$ : Flow rate per unit blade width $\mathrm{m}^{3} / \mathrm{mm} \cdot \mathrm{s}$

Q: Flow rate $\mathrm{m}^{3} / \mathrm{s}$

T: Torque N.m

$u$ : Circumferential velocity $\mathrm{m} / \mathrm{s}$

$v$ : Absolute velocity $\mathrm{m} / \mathrm{s}$ 
VF: Volume fraction of water

$w$ : Relative velocity $\mathrm{m} / \mathrm{s}$

\section{Greek Letters}

$\beta$ : Relative flow angle ${ }^{\circ}$

$\beta_{b}$ : Blade angle ${ }^{\circ}$

$\eta$ : Turbine efficiency $(=P / \rho g Q H)$

$\theta$ : Circumferential angle ${ }^{\circ}$

$\rho:$ Density of water $\mathrm{kg} / \mathrm{m}^{3}$

$w$ : Water area

\section{Subscripts}

1: Runner inlet

2: Runner outlet

3: Upstream

4: Downstream

$a$ : Axial component

$h$ : Hub

$r$ : Radial component

$t$ : Tip

$u$ : Circumferential component.

\section{Conflicts of Interest}

The authors declare that they have no conflicts of interest regarding the publication of this paper.

\section{Acknowledgments}

The authors acknowledge the support of Shinoda Co., Ltd. in the design and production of the experimental apparatus. They are also grateful to Tomoaki Tanemura and Kentaro Hatano, graduate students of Ibaraki University at the time, who supported us with the experiments and numerical analysis. Here, we express our sincere gratitude for their cooperation.

\section{References}

[1] C. Nicolet, A. Zobeiri, P. Maruzewski, and F. Avellan, "Experimental investigations on upper part load vortex rope pressure fluctuations in francis turbine draft tube," International Journal of Fluid Machinery and Systems, vol. 4, no. 1, pp. 179-190, 2011.

[2] T. Vu, M. Koller, M. Gauthier, and C. Deschenes, "Flow simulation and efficiency hill chart prediction for a Propeller turbine," International Journal of Fluid Machinery and Systems, vol. 4, no. 2, pp. 243-254, 2011.

[3] Y. Nishi, T. Inagaki, K. Okubo, and N. Kikuchi, "Study on an axial flow hydraulic turbine with collection device," International Journal of Rotating Machinery, vol. 2014, Article ID 308058, 11 pages, 2014.

[4] A. Georgescu, S. Georgescu, C. I. Cosoiu, and N. Alboiu, "Efficiency of marine hydropower farms consisting of multiplevertical axis cross-flow turbines," International Journal of Fluid Machinery and Systems, vol. 4, no. 1, pp. 150-160, 2011.

[5] M. Anyi and B. Kirke, "Evaluation of small axial flow hydrokinetic turbines for remote communities," Energy for Sustainable Development, vol. 14, no. 2, pp. 110-116, 2010.
[6] A. Inagaki and T. Kanemoto, "Performance of gyro-type hydraulic turbine suitable for shallow stream," Turbomachinery, vol. 33, no. 10, pp. 614-621, 2005.

[7] M. Nakajima, S. Iio, and T. Ikeda, "Performance of savonius rotor for environmentally friendly hydraulic turbine," Journal of Fluid Science and Technology, vol. 3, no. 3, pp. 420-429, 2008.

[8] F. Zotlöterer, "Hydroelectric power plant," Patent WO 2004/ 061295 A3, 2004.

[9] S. Wanchat, R. Suntivarakorn, S. Wanchat, K. Tonmit, and P. Kayanyiem, "A parametric study of a gravitation vortex power plant," Advanced Materials Research, vol. 805-806, pp. 811-817, 2013.

[10] S. Dhakal, S. Nakarmi, P. Pun, A. B. Thapa, and T. R. Bajracharya, "Development and testing of runner and conical basin for gravitational water vortex power plant," Journal of the Institute of Engineering, vol. 10, no. 1, pp. 140-148, 2014.

[11] H. M. Shabara, O. B. Yaakob, Y. M. Ahmed, A. H. Elbatran, and M. S. M. Faddir, "CFD validation for efficient gravitational vortex pool system," Jurnal Teknologi, vol. 74, no. 5, pp. 97-100, 2015.

[12] C. Power, A. McNabola, and P. Coughlan, "A parametric experimental investigation of the operating conditions of gravitational vortex hydropower (GVHP)," Journal of Clean Energy Technologies, vol. 4, no. 2, pp. 112-119, 2015.

[13] J. Matsui, "Internal flow and performance of the spiral water turbine," Turbomachinery, vol. 38, no. 6, pp. 358-364, 2010.

[14] Y. Nishi, T. Inagaki, Y. Li, R. Omiya, and K. Hatano, "The flow field of undershot cross-flow water turbines based on PIV measurements and numerical analysis," International Journal of Fluid Machinery and Systems, vol. 7, no. 4, pp. 174-182, 2014.

[15] Y. Nishi, T. Inagaki, Y. Li, and K. Hatano, "Study on an undershot cross-flow water turbine with straight blades," International Journal of Rotating Machinery, vol. 2015, Article ID 817926, 10 pages, 2015.

[16] N. Kolekar and A. Banerjee, "Performance characterization and placement of a marine hydrokinetic turbine in a tidal channel under boundary proximity and blockage effects," Applied Energy, vol. 148, pp. 121-133, 2015.

[17] J. Riglin, W. Chris Schleicher, I.-H. Liu, and A. Oztekin, "Characterization of a micro-hydrokinetic turbine in close proximity to the free surface," Ocean Engineering, vol. 110, pp. 270-280, 2015

[18] C. W. Hirt and B. D. Nichols, "Volume of fluid (VOF) method for the dynamics of free boundaries," Journal of Computational Physics, vol. 39, no. 1, pp. 201-225, 1981.

[19] F. R. Menter, "Two-equation eddy-viscosity turbulence models for engineering applications," AIAA journal, vol. 32, no. 8, pp. 1598-1605, 1994. 


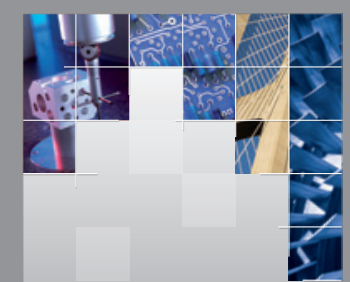

\section{Enfincering}
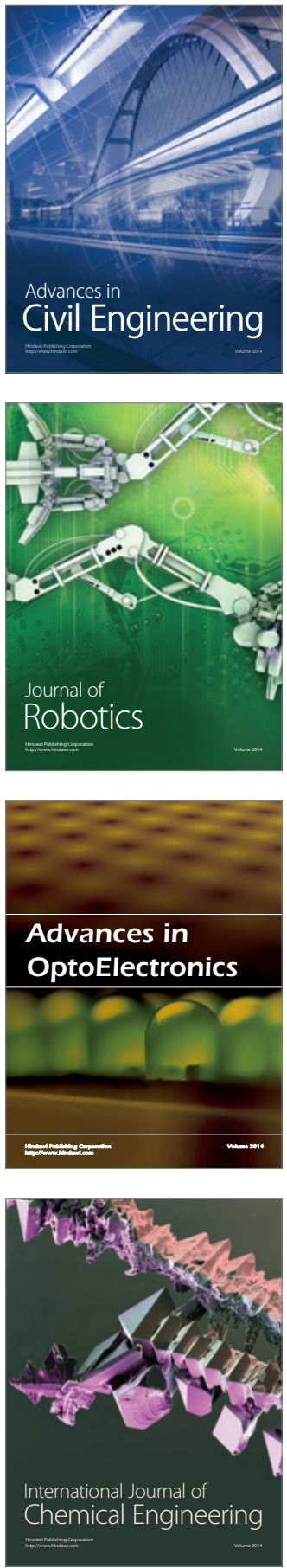

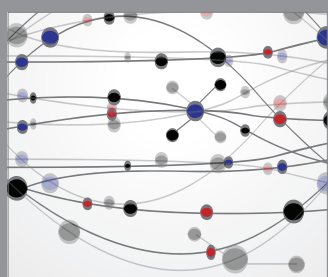

The Scientific World Journal

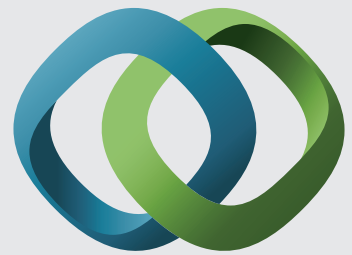

\section{Hindawi}

Submit your manuscripts at

https://www.hindawi.com
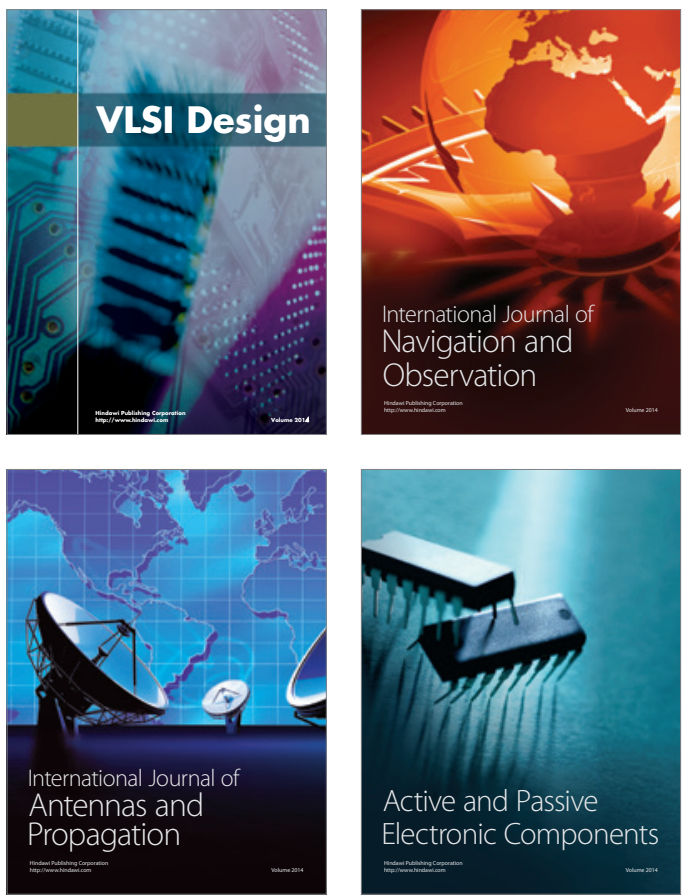
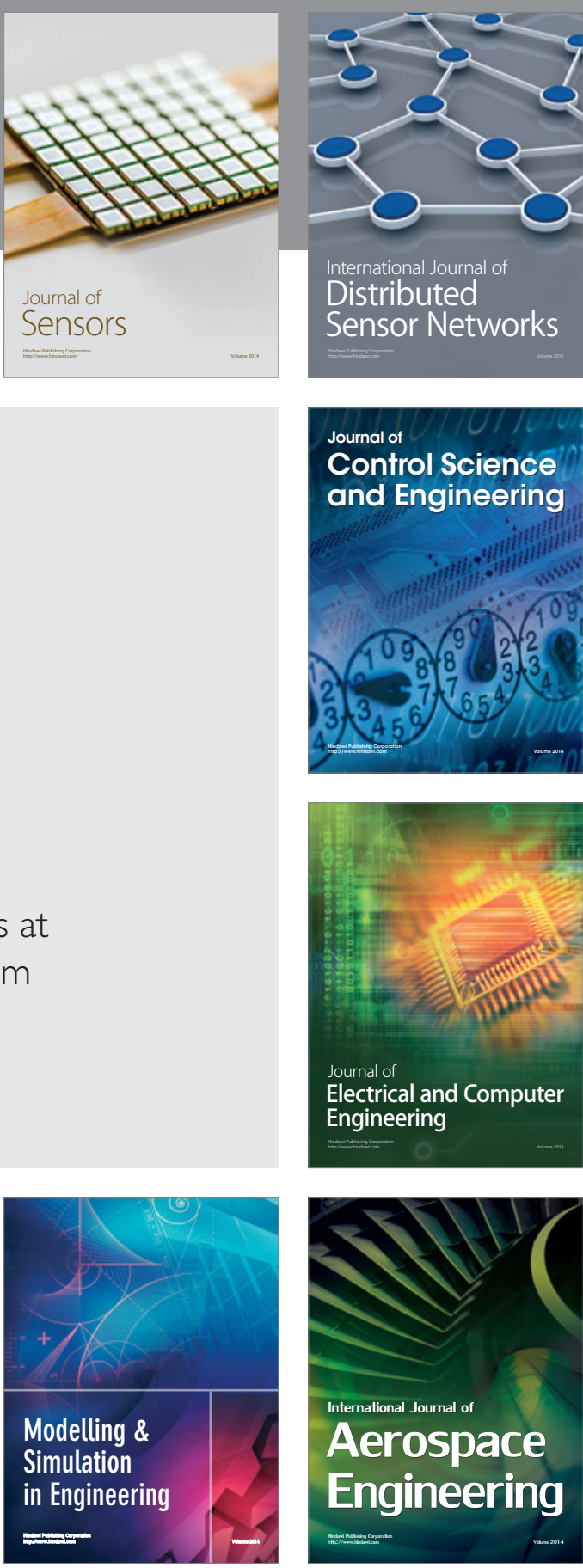

International Journal of

Distributed

Sensor Networks

$-$

Joumal of

Control Science

and Engineering
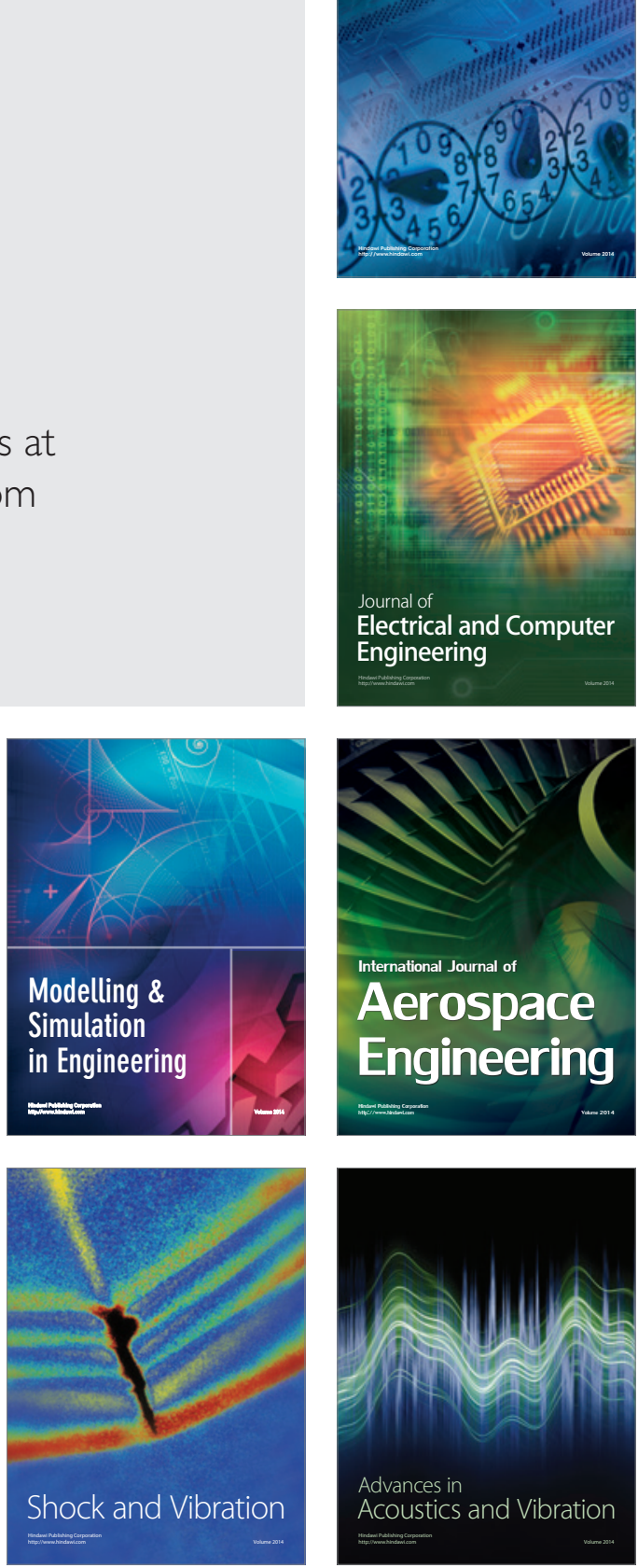